\title{
Direct Observation of the Ultrafast Exciton Dissociation in Lead-iodide Perovskite by 2D Electronic Spectroscopy
}

Ajay Jha ${ }^{1, *}$, Hong-Guang Duan ${ }^{1,2,3, *}$, Vandana Tiwari ${ }^{1,4}$, Pabitra K. Nayak ${ }^{5}$, Henry J. Snaith ${ }^{5}$, Michael Thorwart ${ }^{2,3}$ \& R. J. Dwayne Miller ${ }^{1,3,6}$

${ }^{1}$ Max Planck Institute for the Structure and Dynamics of Matter, Luruper Chaussee 149, 22761, Hamburg, Germany

${ }^{2}$ I. Institut für Theoretische Physik, Universität Hamburg, Jungiusstraße 9, 20355 Hamburg, Germany

${ }^{3}$ The Hamburg Center for Ultrafast Imaging, Luruper Chaussee 149, 22761 Hamburg, Germany

${ }^{4}$ Department of Chemistry, University of Hamburg, Martin-Luther-King Platz 6, 20146 Hamburg, Germany

${ }^{5}$ Department of Physics, University of Oxford, Clarendon Laboratory, Parks Road, Oxford OX1 3PU, United Kingdom

${ }^{6}$ The Departments of Chemistry and Physics, University of Toronto, 80 St. George Street, Toronto Canada M5S $3 \mathrm{H6}$

${ }^{*}$ These authors contributed equally to this work.

September 1, 2017

The unprecedented success of hybrid organic-inorganic lead halide perovskites in photovoltaics motivates fundamental research to unravel the underlying microscopic mechanism for the photoinduced charge generation. Recent studies suggest that most photo-excitations 
in perovskites are free charge carriers, although the contribution of the electron-hole pairs (i.e. excitons) at room temperature has been a matter of debate. We have employed ultrafast two-dimensional (2D) electronic spectroscopy to directly probe the elementary optical excitation of $\mathrm{CH}_{3} \mathrm{NH}_{3} \mathrm{PbI}_{3}$ thin films with $\sim \mathbf{1 6}$ fs temporal resolution. We distinctly capture the ultrafast dissociation of excitons to the free carriers at room temperature and at $180 \mathrm{~K}$. Interestingly, we also observe that the coherent oscillations of the off-diagonal signals in the 2D electronic spectra live for $\sim 50$ fs at room temperature. The entropy-driven dissociation of excitons to free carriers happens within the electronic dephasing timescale and is favored by the low exciton binding energy which we determine to be $\sim 12 \mathrm{meV}$ at room temperature. This ultrafast dissociation of excitons to free carriers can be one of the crucial reasons behind the high efficiency of perovskite based photovoltaics.

\section{Introduction}

Hybrid lead halide perovskites (LHP) are known to have facile solution processability ${ }^{1}$ and reasonably high charge carrier mobility. ${ }^{2,3}$ Therefore, they have become an attractive emerging material for solar cell, photodetector and lasing applications. ${ }^{4-10}$ Since Miyasaka and coworkers first realized perovskite-sensitized solar cells with a power conversion efficiency (PCE) of $3.8 \%^{11}$ in 2009 and the achievement of highly efficient solid state perovskite cells in $2012^{7}$, the perovskite based photovoltaics has shown phenomenally rapid rise in PCE. With improved deposition methods and rational selection of device designs, the PCE has meteorically advanced to $22.1 \%$ based on a solid polycrystalline perovskite absorber layer sandwiched between charge selective contacts. ${ }^{4,12-14}$ For 
further rational tailoring of the device efficiency and stability, it is imperative to actively pursue the fundamental studies of underlying photophysical mechanisms for charge generation.

To advance the understanding of the basic photophysical processes, significant experimental and theoretical efforts have been expended focusing on different aspects of the problem. ${ }^{3,15-18,30}$ Recent studies have converged on the proposal that most elementary photoexcitations in perovskite are free charge carriers, which behave like III-V inorganic semiconductors. In addition, the significant role of bound electron-hole pairs, or excitons, has been realized. ${ }^{19-22}$ These sub-bandgap excitonic bound states govern key processes such as charge transport and light emission and are thus the determining factors for the performance of any optoelectronic device. ${ }^{23-26}$ In perspective of photovoltaics, the coupling of the excitons to the bath and their subsequent dissociation into free charge carriers are the central important processes in device photophysics. The exciton binding energy $\left(E_{\mathrm{b}}\right)$ is one of the important parameter that dictates exciton dissociation and hence, the determination of $E_{\mathrm{b}}$ at room temperature is crucial for accessing and further development of any material for photovoltaics. ${ }^{31}$ For LHP, a wide range of exciton binding energies from 2 to $55 \mathrm{meV}$ have been reported using steady state absorption, photocurrent, photoluminescence and electroabsorption spectroscopies. ${ }^{32-37}$ In a recent study, using magneto-optics measurements, $E_{\mathrm{b}}$ at low temperature has been determined to be $\sim 16 \mathrm{meV}{ }^{35}$ The value at room temperature could not be determined by magneto optics measurements due to thermal broadening. The photoluminescence spectrum of the LHP shows a narrow exciton peak at low temperature, which indicates quite weak exciton-phonon interactions in this material. ${ }^{32}$ In addition to $E_{\mathrm{b}}$, the dynamics of the exciton is also determined by the exciton-phonon coupling. ${ }^{27-29}$ Recent studies suggest that the 
exciton screening by the collective reorientation motion of the organic cations at room temperature weakens this interaction. ${ }^{38-41}$ To gather a comprehensive understanding of the exciton dynamics at room temperature, it is imperative to directly observe the exciton. The free-exciton peak has been well-resolved at low temperature by the photocurrent, photoluminescence ${ }^{32}$ and four-wave mixing spectroscopy. ${ }^{42}$ However, at elevated temperature, the exciton peak shifts and is lost within the broad bandwidth of the free carrier transitions, which makes the detection of free excitons challenging. Despite enormous experimental and theoretical efforts, there has been no conclusive report of the direct generation of free excitons after photoexcitation in LHP at room temperature.

Two-dimensional (2D) electronic spectroscopy is one of the effective tools, which allows for direct mapping of the electronic dynamics as a function of the absorption and emission wavelength. ${ }^{44}$ It is particularly useful for examining systems with manifolds of electronic states, i.e., solidstate materials with band structures, and of systems where static disorder induces strong spectral broadening and highly congested spectra. It has been successfully implemented to reveal the excitation energy transfer pathways in the photosynthetic protein complex ${ }^{45-48}$ and in inorganic semiconductors. ${ }^{49,50}$ Here, we use $2 \mathrm{D}$ electronic spectroscopy to capture the electronic dynamics of sub-bandgap bulk excitons after optical excitation of a LHP thin film at room temperature and $180 \mathrm{~K}$. The high temporal resolution of 16 fs allows us to discriminate exciton and free carrier transitions and resolve the exciton binding energy of $12 \mathrm{meV}$ at room temperature. Moreover, we observe the ultrafast dissociation dynamics of the free excitons within the electronic dephasing time scale of $\sim 50$ fs at $296 \mathrm{~K}$, resulting in primarily free charge carrier population after this process. 


\section{Results and Discussion}

Thin film of $\mathrm{CH}_{3} \mathrm{NH}_{3} \mathrm{PbI}_{3}$ perovskite has been prepared over a $2 \mathrm{~mm}$ thick quartz substrate. The details for the sample preparation and its characterization have been discussed in the materials and methods section. Figure 1(a) represents the absorption spectrum (red line) of the perovskite film in a spectral range between $12000 \mathrm{~cm}^{-1}$ to $15000 \mathrm{~cm}^{-1}$ at room temperature. The absorption grows steeply with photon energy starting at $12900 \mathrm{~cm}^{-1}$. The peak at $13550 \mathrm{~cm}^{-1}$ corresponds to the bandgap transition from the valence band (VB) to the conduction band (CB). The bandgap energy $\left(E_{\mathrm{g}}\right)$ of $\mathrm{CH}_{3} \mathrm{NH}_{3} \mathrm{PbI}_{3}$ perovskite has been reported to be $13200 \mathrm{~cm}^{-1}$, marked as a blue dashed

line in Figure 1(a). ${ }^{52,53}$ The feature corresponding to excitonic transition is completely submerged within the conduction band continuum absorption envelope. The $\sim 16$ fs excitation laser spectrum used for the 2D electronic measurements is shown as blue shaded region in Figure 1(a). The frequency window of the laser spectrum $\left(12000 \mathrm{~cm}^{-1}\right.$ to $\left.14500 \mathrm{~cm}^{-1}\right)$ is sufficiently broad to cover the bandgap transition as well as the proposed excitonic transition (red arrow in Figure 1(b)).

Two-dimensional electronic spectroscopy provides correlation maps between the excitation $\left(\omega_{t}\right)$ and the probing wavelength $\left(\omega_{\tau}\right)$, in contrast to pump probe spectroscopy, which does not provide resolution on the excitation frequency. ${ }^{44}$ These correlation maps envisage connectivity in different electronic transitions with high temporal and spectral resolution. To decipher the interplay of excitonic and bandgap transitions in $\mathrm{CH}_{3} \mathrm{NH}_{3} \mathrm{PbI}_{3}$ perovskite, we measured a series of photonecho $2 \mathrm{D}$ electronic spectra at room temperature $(296 \mathrm{~K})$ and $180 \mathrm{~K}$ for different waiting times. The details of the experimental set up and measurement conditions have been described in the 
materials and method section. The measured $2 \mathrm{D}$ spectrum at $296 \mathrm{~K}$ for the waiting time $T=0 \mathrm{fs}$ is shown in Figure 2(a). The 2D correlation map at $T=0 \mathrm{fs}$ is extremely rich and dominated by a strong bleach signal along the diagonal with the presence of absorption signals as off-diagonal features. The resonant bleach signature corresponds to generation of free carriers in LHP. This peak is significantly stretched along the diagonal direction which manifests a strong inhomogeneous broadening of the free-carrier transition. Moreover, one additional diagonal peak as shoulder is well resolved at $\omega_{\tau}=13100 \mathrm{~cm}^{-1}$, although with a lower intensity. For better visualization of this feature, a cut along the diagonal of the $2 \mathrm{D}$ spectrum at $T=0$ fs is plotted in Figure 2(b). In addition to the resonant features corresponding to different eigenstates of the bandgap transition (shown as blue and magenta sticks), the sub-bandgap peak at $13100 \mathrm{~cm}^{-1}$ (marked as red stick in Figure 2(b)) is clearly resolved as a shoulder to the central bleach band. This peak is in good agreement with the excitonic transition resolved by the Elliott model, which lies slightly below the free-carrier transition as shown in Figure1(b). ${ }^{34,36,51-53}$ Hence, we assign the peak at $13100 \mathrm{~cm}^{-1}$ to the excitonic transition. Since we can observe the excitonic level, this enables us to determine the exciton binding energy $E_{\mathrm{b}}$ of the system. As shown in Figure1(b), $E_{\mathrm{b}}$ is the difference in energy between the exciton and continuous transition. Using our observed value of the excitonic transition $\left(13100 \mathrm{~cm}^{-1}\right)$ and the reported bandgap energy value $\left(13200 \mathrm{~cm}^{-1}\right),{ }^{52,53}$ exciton binding energy has been obtained to be $\sim 12 \mathrm{meV}$. Our value for $E_{\mathrm{b}}$ is in the range of reported values of 5 to $55 \mathrm{meV}$ from the previous measurements. Unlike the other approaches, the 2D spectroscopy allows us to directly observe the resonant excitonic transition just after photoexcitation in LHP. In addition to the strong diagonal features, the off-diagonal peaks have negative magnitude in the 
2D spectrum at $T=0$ fs. These features possibly come from the excited-state absorption of the free-carrier band, along with a small contribution originating from the substrate (see Supporting Information for the details of the substrate and signal contribution, Figure S2). Thus, based on the observation of the 2D spectrum at $T=0 \mathrm{fs}$, we have clearly demonstrated that the free carriers and the excitons are simultaneously generated in LHP after the photo-excitation at room temperature, which is hard to be realized by other spectroscopic techniques. ${ }^{33}$

The 2D correlation map at $T=0$ fs manifests the information about the fundamental lineshape of the transitions under study and hence, can be used to extract respective time scales of electronic dephasing. ${ }^{54}$ The lifetime of the electronic coherence generated by the coherent laser excitation is mainly determined by the electron-phonon and exciton-phonon interactions in LHP. Thus, the anti-diagonal bandwidth can be used as a reporter to compare the strengths of these interactions. For a quantitative analysis, we fit the profile along the anti-diagonal direction for both peaks to Lorentzian lineshapes as shown in Figure 2(c) and (d). We obtain the lifetimes of $41 \mathrm{fs}$ and $47 \mathrm{fs}$ for the electronic dephasing of the free carriers and the excitons, respectively. Interestingly, it is comparable to the value of a rather small solvated cyanine dye molecule. ${ }^{54}$ Hence, the electron-phonon and exciton-phonon interactions in LHP thin film can be considered as quite weak. This is probably caused by the protection of the free carriers and excitons in LHP by charge screening. ${ }^{38}$

In order to track the fate of the observed excitons at $13100 \mathrm{~cm}^{-1}$ in $\mathrm{CH}_{3} \mathrm{NH}_{3} \mathrm{PbI}_{3}$, we have analysed the 2D spectra at different waiting times after photoexcitation. Figures 3(a), (b) and (c) 
represent the $2 \mathrm{D}$ spectrum obtained at the waiting times $T=20,50$ and $200 \mathrm{fs}$, respectively. At $T=20 \mathrm{fs}$, the $2 \mathrm{D}$ spectrum is significantly shrinked along its diagonal direction which indicates a dramatically reduced inhomogeneous broadening. This is due to the loss of initial correlations between excitation and the detection window as the time progresses and is known as spectral diffusion. In addition to the changes in the diagonal features, the excited-state absorption (blue peaks) shows separated cross peaks above $13500 \mathrm{~cm}^{-1}$, which possibly indicates the absorption from different states of the continuum band. The magnitude of the exciton peak at $13100 \mathrm{~cm}^{-1}$ significantly decays for increasing waiting times and is hard to be observed at $T=50 \mathrm{fs}$, as can be seen in Figure 3(b). Meanwhile, one new cross peak appears at $\left(\omega_{\tau}, \omega_{t}\right)=\left(13550 \mathrm{~cm}^{-1}, 12950 \mathrm{~cm}^{-1}\right)($ marked by $\mathrm{C}$ in Figure 3(b)), although it strongly overlaps with the diagonal peak at $\omega_{\tau}=13550 \mathrm{~cm}^{-1}$. This cross peak $\mathrm{C}$ is the evidence of an electronic coupling between the exciton and the free carriers. To gain insight to the population dynamics, we extract the time evolution traces of the selected peaks $\mathrm{C}$ and $\mathrm{D}$ marked in the $2 \mathrm{D}$ spectrum at $T=50 \mathrm{fs}$ in Figure 3(b). The kinetic traces corresponding to these off-diagonal cross-peaks are shown in Figure 3(d). We observe that the magnitude of the cross peak C (red line) dramatically increases and reaches its maximum within 50 fs. Interestingly, the rise of the cross peak is complemented by the observed decay of the excitonic band at 13100 $\mathrm{cm}^{-1}$. Thus, we can conclude that photo-generated excitons undergo an ultrafast dissociation to free carriers. Moreover, the time-dependent trace of the second off-diagonal peak (marked by a blue line in Figure 3(d)) shows evidence of oscillations within the initial time window of $50 \mathrm{fs}$, which also matches with the timescale of electronic dephasing extracted from the anti-diagonal bandwidth discussed in Figure 2(c) and (d). To explore the temperature dependence of the exci- 
ton dissociation process, we measured the $2 \mathrm{D}$ spectra of a $\mathrm{CH}_{3} \mathrm{NH}_{3} \mathrm{PbI}_{3}$ thin film at $180 \mathrm{~K}$. The excitonic peak is better separated from the bandgap absorption at low temperature, as shown in the supporting information, Figure S5. The kinetic traces of the cross peaks C and D at $180 \mathrm{~K}$ are shown in Figure 3(d) as green and magenta dashed lines, respectively. They show similar feature of the population transfer within the initial $50 \mathrm{fs}$ (green dashed line) and the short-lived oscillations (magenta dashed line), as has also been observed at room temperature. To further resolve the observed oscillations, a Fourier transform analysis has been performed which shows a broadband peak centered at $750 \mathrm{~cm}^{-1}$ (see supporting information, Figure S6). Additionally, the central peak in the $2 \mathrm{D}$ spectra for $T=50 \mathrm{fs}$ has decayed further and the magnitude of the off-diagonal peaks corresponding to the excited-state absorption is significantly reduced, as shown in Figure 3(b). At $T=200$ fs, we do not observe excited-state absorption in the free-carrier region nor is there any evidence of significant inhomogeneous broadening. Instead, the cross peak between the exciton and the free carriers can be seen more clearly as a stretch along $\omega_{t}$ in the $2 \mathrm{D}$ spectrum.

To ensure that the exciton decay has no contribution from exciton-exciton scattering, the energy of the pump beam has been tuned to generate the excitons well below the Mott density (more details are given in the supporting information). The kinetics of the exciton and free-carrier peaks are extracted from the 2D spectra and shown in Figure 4(a) and (b), respectively (marked by $A$ and $B$ in the 2D spectra at $T=0 \mathrm{fs}$ in Figure 2(a)). Unlike free carriers, excitons decay independently of the pump energy which signifies the linear regime for the exciton dynamics. The exponential decay of the exciton peak with a 30 fs time constant shows that the free excitons undergo an ultrafast dissociation process to free carriers. In contrast to the exciton decay, the 
decay rates of the free carriers show strong dependence on the pump power, as shown in Figure 4(b). The observed increased rate of recombination of free carriers at higher intensities is caused by second-order non-geminate recombination process. ${ }^{30}$ Thus, the decay of the exciton to free carriers happens on an ultrafast timescale which has been observed to be similar to the electronic dephasing timescale. This unusual energetic uphill transition can possibly be explained as an electronic process which is entropically driven by the electronically coupled much higher density of states in the conduction band. For the analogy of this process, $\mathrm{Zu}$ and co-workers also observed coherent electronic coupling in tetracene molecules which overcome the energy barrier in singlet fission. ${ }^{55}$ To verify this, we compared the kinetic traces of the exciton peak at room temperature and $180 \mathrm{~K}$ (as shown in Figure 4(c)). We observe that the rate of exciton dissociation is not sensitive to the temperature factor, which is strongly related to acoustic phonons in the lattice. This temperature-independent dissociation validates our understanding of entropically driven ultrafast exciton decay.

To gain further insight, we have generated the three-dimensional matrix of time-evolved 2D electronic spectra and analyzed the kinetics by the global fitting approach. ${ }^{56}$ Additionally, we have also performed the correlation analysis (see the supplementary information for details) of the residuals obtained by subtracting the underlying global kinetics from the measured data. This analysis provides the $2 \mathrm{D}$ correlation map as shown in the supporting information, Figure $\mathrm{S} 4$. We observe that the cross peaks in the correlation map can generally be divided into two parts along the diagonal direction. First, many cross peaks are present above the frequency $13500 \mathrm{~cm}^{-1}$ with a negative magnitude, which clearly shows evidence of the associated oscillations during the free-carrier dy- 
namics. The cross peaks are marked by the black dotted lines to illustrate the possibility of related vibrational levels in the conduction band. Secondly, two cross peaks are clearly resolved with positive magnitude (although being weak), which corresponds to the correlation of the exciton and free-carrier band. Earlier reports from different groups have shown that the positive correlation in this correlation analysis provides evidence of electronic coherence. ${ }^{48,57}$ Thus, based on this correlation analysis and the timescale of the electronic dephasing, our measurements on $\mathrm{CH}_{3} \mathrm{NH}_{3} \mathrm{PbI}_{3}$ perovskite hint towards the possible role of electronic coherence in ultrafast exciton dissociation into free carriers. However, the short lifetime of the coherence along with the small magnitude of the exciton signal renders it extremely hard for us to ascertain the functional role of the electronic coherence. Thus, we cannot exclude the possibility that the energy-uphill transition of the exciton to the free carriers is barely driven by entropy which is facilitated by the electronically coupled much higher density of states in the conduction band.

In order to resolve the relative contributions of the vibrational frequencies that produce the beating in the 2D spectra, a Fourier transform of the residuals from the global analysis has been performed (see the supplementary information for details). In order to show the signal-to-noise ratio, the power spectrum in the particular region of the free-carrier band is plotted in Figures 4(d), (e) and (f). To visualize these vibrational modes, we have plotted the $2 \mathrm{D}$ vibrational maps as shown in the supporting information, Figure S7. In addition to a strong peak at $190 \mathrm{~cm}^{-1}$, two low-frequency vibrational modes at 48 and $96 \mathrm{~cm}^{-1}$ are clearly resolved. Based on the reported quantum calculations, ${ }^{59,60}$ they are assigned to the "breathing" modes of the inorganic octrahedra, which have been suggested as the central important modes related to the charge-carrier 
dynamics in LHP. ${ }^{61}$ In the light of the weak electron-phonon coupling in perovskite, the role of these lattice modes should be revised using other spectroscopic techniques. Finally, a few highfrequency modes associated with $\mathrm{CH}_{3} \mathrm{NH}_{3}^{+}$have also been observed which are primarily centered in the conduction band (see the supplementary information) and are in agreement with the reported literature. ${ }^{60,62}$

\section{Conclusions}

In conclusion, we have established direct spectroscopic evidence for the co-existence of excitons and free carriers in the LHP after photo-excitation at room temperature and $180 \mathrm{~K}$ using $2 \mathrm{D}$ electronic spectroscopy. Direct observation of the exciton after photoexcitation allows us to obtain the binding energy of $12 \mathrm{meV}$ for LHP at room temperature. This shows that the binding energy $\mathrm{E}_{\mathrm{b}}$ does not completely collapse at room temperature, but retains a similar value to that determined at lower temperatures. ${ }^{35}$ Additionally, the high temporal resolution enables us to capture the ultrafast decay of free excitons to the charge carriers occurring in a time span of $50 \mathrm{fs}$. Based on the correlation analysis, we show that this entropically driven uphill transition is probably favored by the coherence between excitonic and conduction band of states. The timescales of the electronic dephasing, $41 \mathrm{fs}$ and $47 \mathrm{fs}$, have been resolved by measuring the anti-diagonal bandwidth of the 2D spectrum at zero waiting time. This indicates the relatively weak exciton-phonon and electronphonon interaction in the LHP. Moreover, it indirectly provides indication that excitons and electrons in LHP are possibly protected by charge screening effects. ${ }^{39}$ This possibly provides a degree of immunity to mid-gap state trapping and associated non-radiative decay losses. Our results pro- 
vide important fundamental insight into the nature of photo-generated species at the band-edge of LHP which elucidates the photophysical mechanisms underlying the remarkable performance of this material in optoelectronic devices.

\section{Matierials and methods}

Sample preparation. The precursor solution for the perovskite film was prepared by dissolving 442.2 mg of methylammonium iodide (Dyesol) and $351.2 \mathrm{mg}$ of lead(II)acetate trihydrate (SigmaAldrich) in $4 \mathrm{ml}$ of anhydrous $\mathrm{N}, \mathrm{N}$-dimethylformamide (Sigma-Aldrich). The perovskites films were prepared by spin-coating the precursor solution at 2000 r.p.m on quartz substrates for $45 \mathrm{~s}$ under a nitrogen atmosphere in a glove box. Then, the films were left for drying for 10 minutes followed by annealing at $100{ }^{\circ} \mathrm{C}$ for 5 minutes in the glove box. The UV-Vis spectrum of the thin film was taken on a Carry $300 \mathrm{UV}$-Vis spectrometer. The steady-state photoluminescence measurement was done on a Flourolog Horiba Jobin Yvon. A scanning electron microscope image was taken on a Hitachi S-4300 (see Supporting Information for the characterization data).

Experimental setup and measuring conductions. Ultrashort coherent pulses were generated by a home-built nonlinear optical parametric amplifier pumped by a commercial femtosecond laser Pharos (Light Conversion). A broadband spectrum with a linewidth of 100 nm (FWHM) was centered at $13300 \mathrm{~cm}^{-1}$ such that an overlap with the near infrared region of the absorption spectrum of Perovskites is achieved. The excitation pulse was further compressed to the Fourier transformlimited with duration of 16 fs by the combination of a prism pair (F2) and a deformable mirror 
(OKO Technologies). Their temporal profiles were characterized by means of frequency-resolved optical grating (FROG). The measured FROG traces were analyzed using a commercial program FROG3 (Femtosecond Technologies). The 2D spectra were collected in an all-reflective 2D spectrometer based on a diffractive optic (Holoeye) with a phase stability of $\lambda / 160$ whose configuration is described elsewhere ${ }^{63}$. Further components were the Sciencetech spectrometer model 9055 and a high-sensitive CCD linear array camera (Entwicklungsburo Stresing). The 2D spectra were collected at each fixed waiting time $\mathrm{T}$ by scanning the delay time $\tau=t_{1}-t_{2}$ in the range of $[-128 \mathrm{fs}, 128 \mathrm{fs}]$ with a delay step of $1 \mathrm{fs}$. At each delay point, 200 spectra were averaged to improve the signal to noise ratio. The waiting time $T=t_{3}-t_{2}$ was linearly scanned in the range of $0-2 \mathrm{ps}$ in steps of $10 \mathrm{fs}$, and with a logarithmically spaced delay time-step up to $100 \mathrm{ps}$. The energy of excitation pulse is limited to $18 \mathrm{~nJ}$ with $1 \mathrm{KHz}$ repetition rates for room-temperature measurements. To check the pump fluence dependence, we also carried out measurements with different pump energies (see Fig. 4(a), (b) and Supporting Information for details). Three pulses are focused on the sample with the spot size $100 \mu \mathrm{m}$ and the photon echo signal is generated in the phase-matching direction. To avoid oxidation, the sample was kept in the cryostat (MicrostatHe2 (Rect. Tail) from Oxford Instruments) under vacuum condition $\left(1.7 \times 10^{-6} \mathrm{~Pa}\right)$. To check for reproducibility of the results, measurements are performed on different spots on the perovskite films as well as films prepared from three different batches which showed similar spectral features.

Data Analysis. 2D spectra are obtained from the measured photon-echo (PE) signals according to an established earlier reported procedure. ${ }^{44}$ Briefly, the PE-traces extracted from the heterodyne signals were transformed along the delay axis $\tau$ in a whole measuring range (which results in 
a frequency axis $\omega_{\tau}$ ) using numerical Fourier transform. The spectra along the observation axis which corresponds to the wavelength scale in the spectrometer were interpolated to the equallyspaced frequencies $\omega_{t}$. Phasing of the obtained 2D spectra, i.e., finding the correct delay of the local oscillator (LO)-filter used, was performed using an "invariant theorem". ${ }^{44}$ It states that the real parts of both heterodyne-detected PE and transient grating (TG) spectra are equal at zero delay time, $\tau$ to the pump-probe (PP) spectrum measured at the same waiting time, $T$. We perform phasing of the PE heterodyne signal based on the PP signal at the particular waiting time. The stability of the measurement can be examined by the LO delay.

1. Burschka, J.; Pellet, N.; Moon, S. -J.; Humphry-Baker, R.; Gao, P.; Nazeeruddin, M. K.; Grätzel, Mi. Sequential Deposition as a Route to High-performance Perovskite-sensitized Solar Cells. Nature 2013, 499, 316-319.

2. Stranks, S. D.; Eperon, G. E.; Grancini, G.; Menelaou, C.; Alcocer, M. J. P.; Leijtens, T.; Herz, L. M.; Petrozza, A.; Snaith, H. J. Electron-Hole Diffusion Lengths Exceeding 1 Micrometer in an Organometal Trihalide Perovskite Absorber. Science 2013, 342, 341-344.

3. Xing, G.; Mathews, N.; Sun, S.; Lim, S. S.; Lam, Y. M.; Grätzel, M.; Mhaisalkar, S.; Sum, T. C. Long-Range Balanced Electron- and Hole-Transport Lengths in Organic-Inorganic $\mathrm{CH}_{3} \mathrm{NH}_{3} \mathrm{PbI}_{3}$. Science 2013, 342, 344-347.

4. Yang, W. S.; Noh, J. H.; Jeon, N. J.; Kim, Y. C.; Ryu, S.; Seo, J.; Seok, S. I. High-performance Photovoltaic Perovskite Layers Fabricated Through Intramolecular Exchange. Science 2015, $348,1234-1237$. 
5. Zhu, H.; Fu, Y.; Meng, F.; Wu, X.; Gong, Z.; Ding, Q.; Gustafsson, M. V.; Trinh, M. T.; Jin, S.;

Zhu, X. -Y. Lead Halide Perovskite Nanowire Lasers with Low Lasing Thresholds and High Quality Factors. Nat. Mater. 2015, 14, 636-642.

6. Liu, M.; Johnston, M. B.; Snaith, H. J. Efficient Planar Heterojunction Perovskite Solar Cells by Vapour Deposition. Nature 2013, 501, 395-398.

7. Lee, M. M.; Teuscher, J.; Miyasaka, T.; Murakami, T. N.; Snaith, H. J. Efficient Hybrid Solar Cells Based on Meso-Superstructured Organometal Halide Perovskites. Science 2012, 338, 643-647.

8. Kim, H. -S.; Lee, C. -R.; Im, J. -H.; Lee, K. -B.; Moehl, T.; Marchioro, A.; Moon, S. J.; Humphry-Baker, R.; Yum, J. -H.; Moser, J. E.; Grätzel, M.; Park, N. -G. Lead Iodide Perovskite Sensitized All-Solid-State Submicron Thin Film Mesoscopic Solar Cell with Efficiency Exceeding 9\%. Sci. Rep. 2012, 2, 591.

9. Etgar, L.; Gao, P.; Xue, Z.; Peng, Q.; Chandiran, A. K.; Liu, B.; Nazeeruddin, M. K.; Grätzel, M. Mesoscopic $\mathrm{CH}_{3} \mathrm{NH}_{3} \mathrm{PbI}_{3} / \mathrm{TiO}_{2}$ Heterojunction Solar Cells J. Am. Chem. Soc. 2012, 134, 17396-17399.

10. Lin, Q.; Armin, A.; Burn, P. L.; Meredith, P. Filterless Narrowband Visible Photodetectors. Nat. Photonics 2015, 9, 687-694.

11. Kojima, A.; Teshima, K.; Shirai, Y.; Miyasaka, T. Organometal Halide Perovskites as VisibleLight Sensitizers for Photovoltaic Cells. J. Am. Chem. Soc. 2009, 131, 6050-6051. 
12. Jeon, N. J.; Noh, J. H.; Yang, W. S.; Kim, Y. C.; Ryu, S.; Seo, J.; Seok, S. I. Compositional Engineering of Perovskite Materials for High-performance Solar Cells Nature 2015, 517, 476480.

13. for record cell efficiencies, see: Best Research-Cell Efficiencies. http://www.nrel.gov/ncpv/images/efficiency-chart.jpg (2016).

14. Ball, J. M.; Lee, M. M.; Heya, A.; Snaith, H. J. Low-temperature Processed Mesosuperstructured to Thin-film Perovskite Solar Cells Ener. Envir. Sci. 2013, 6, 1739-1743.

15. Ponseca Jr, C. S.; Sundströma, V. Direct Monitoring of Ultrafast Electron and Hole Dynamics in Perovskite Solar Cells. Nanoscale, 2013, 8, 6249-6257.

16. Saba, M.; Quochi, F.; Mura, A.; Bongiovanni, G. Excited State Properties of Hybrid Perovskites. Acc. Chem. Res. 2016, 49, 166-173.

17. Stamplecoskie, K. G.; Manser, J. S.; Kamat, P. V. Dual Nature of the Excited State in Organicinorganic Lead Halide Perovskites. Ener. Environ. Sci. 2014, 8, 208-215.

18. Wu, X.; Trinh, M. T.; Niesner, D.; Zhu, H.; Norman, Z.; Owen, J. S.; Yaffe, O.; Kudisch, B. J.; Zhu, X. -Y. Trap States in Lead Iodide Perovskites. J. Am. Chem. Soc. 2015, 137, 2089-2096.

19. Blancon, J. -C.; Tsai, H.; Nie, W.; Stoumpos, C. C.; Pedesseau, L.; Katan, C.; Kepenekian, M.; Soe, C. M. M.; Appavoo, K.; Sfeir, M. Y.; Tretiak, S.; Ajayan, P. M.; Kanatzidis, M. G.; Even, J.; Crochet, J. J.; Mohite, A. D. Extremely Efficient Internal Exciton Dissociation Through Edge States in Layered 2D Perovskites. Science 2017 355, 1288-1292. 
20. Wang, H.; Whittaker-Brooks, L.; Fleming, G. R. Exciton and Free Charge Dynamics of Methylammonium Lead Iodide Perovskites Are Different in the Tetragonal and Orthorhombic Phases. J. Phys. Chem. C 2015, 119, 19590-19595.

21. Wang, W.; Li, Y.; Wang, X.; Lv, Y.; Wang, S.; Wang, K.; Shi, Y.; Xiao, L.; Chen, Z.; Gong, Q. Density-dependent dynamical coexistence of excitons and free carriers in the organolead perovskite $\mathrm{CH}_{3} \mathrm{NH}_{3} \mathrm{PbI}_{3}$. Phys. Rev. B 2016, 94, 140302.

22. Manser, J. S.; Christians, J. A.; Kamat, P. V. Intriguing Optoelectronic Properties of Metal Halide Perovskites. Chem. Rev. 2016, 116, 12956-13008.

23. Ross, J. S.; Klement, P.; Jones, A. M.; Ghimire, N. J.; Yan, J.; Mandrus, D. G.; Taniguchi, T.; Watanabe, K.; Kitamura, K.; Yao, W.; Cobden, D. H.; Xu, X. Electrically Tunable Excitonic Light-emitting Diodes Based on Monolayer WSe2 p-n Junctions. Nat. Nanotech. 2014, 9, 268-272.

24. Koch, S. W.; Kira, M.; Khitrova, G.; Gibbs, H. M. Semiconductor Excitons in New Light. Nat. Mater. 2006, 5, 523-531.

25. Nozik, A. J.; Beard, M. C.; Luther, J. M.; Law, M.; Ellingson, R. J.; Johnson, J. C. Semiconductor Quantum Dots and Quantum Dot Arrays and Applications of Multiple Exciton Generation to Third-Generation Photovoltaic Solar Cells. Chem. Rev. 2010, 110, 6873-6890.

26. Agranovich, V. M.; Basko, D. M.; La Rocca, G. C.; Bassani, F. Excitons and Optical Nonlinearities in Hybrid Organic-inorganic Nanostructures. J. Phys. C. Matt. 1998, 10, 9369-9400. 
27. Wright, A. D.; Verdi, C.; Milot, R. L.; Eperon, G. E.; Pérez-Osorio, M. A.; Snaith, H. J.; Giustino, F.; Johnston, M. B.; Herz, L. M. Electronphonon Coupling in Hybrid Lead Halide Perovskites. Nat. Comm. 2016, 7, 11755.

28. Straus, D. B.; Parra, S. H.; Iotov, N.; Gebhardt, J.; Rappe, A. M.; Subotnik, J. E.; Kikkawa, J. M.; Kagan, C. R. Direct Observation of ElectronPhonon Coupling and Slow Vibrational Relaxation in OrganicInorganic Hybrid Perovskites. J. Am. Chem. Soc. 2016, 138, 1379813801.

29. Phuong, L. Q.; Nakaike, Y.; Wakamiya, A.; Kanemitsu, Y. Free Carriers versus Excitons in $\mathrm{CH}_{3} \mathrm{NH}_{3} \mathrm{PbI}_{3}$ Perovskite Thin Films at Low Temperatures: Charge Transfer from the Orthorhombic Phase to the Tetragonal Phase. J. Phys. Chem. Lett. 2016, 7, 4905-4910.

30. Manser, J. S.; Kamat, P. V. Band Filling with Free Charge Carriers in Organometal Halide Perovskites. Nat. Photonics 2014, 8, 737-743.

31. Scholes, G. D. Insights into Excitons Confined to Nanoscale Systems: Electron-Hole Interaction, Binding Energy, and Photodissociation. ACS Nano 2008, 2, 523-537.

32. Phuong, L. Q.; Nakaike, Y.; Wakamiya, A.; Kanemitsu, Y. Free Carriers versus Excitons in CH3NH3PbI3 Perovskite Thin Films at Low Temperatures: Charge Transfer from the Orthorhombic Phase to the Tetragonal Phase. J. Phys. Chem. Lett. 2016, 7, 2316-2321.

33. D’Innocenzo, V.; Grancini, G.; Alcocer, M. J. P.; Ram Srimath Kandada, A.; Stranks, S. D.; Lee, M. M.; Lanzani, G.; Snaith H. J. Excitons Versus Free Charges in Organo-lead Tri-halide Perovskites. Nat. Comm. 2014, 5, 3586. 
34. Sestu, N.; Cadelano, M.; Sarritzu, V.; Chen, F.; Marongiu, D.; Piras, R.; Mainas, M.; Quochi, F.; Saba, M.; Mura, A.; Bongiovanni, G. Absorption F-Sum Rule for the Exciton Binding Energy in Methylammonium Lead Halide Perovskites. J. Phys. Chem. Lett. 2015, 6, 45664572.

35. Miyata, A.; Mitioglu, A.; Plochocka, P.; Portugall, O.; Wang, J. T.; Stranks, S. D.; Snaith, H. J.; Nicholas, R. J. Direct Measurement of the Exciton Binding Energy and Effective Masses for Charge Carriers in Organic-inorganic Tri-halide Perovskites. Nat. Phys. 2015, 11, 582-587.

36. Yang, Y.; Ostrowski, D. P.; France, R. M.; Zhu, K.; Lagemaat, J.; Luther, J. M.; Beard, M. C. Observation of a Hot-phonon Bottleneck in Lead-iodide Perovskites. Nat. Photonics 2016, 10, 53-59.

37. Ziffer, M. E.; Mohammed, J. C.; Ginger, D. S. Electroabsorption Spectroscopy Measurements of the Exciton Binding Energy, Electron-Hole Reduced Effective Mass, and Band Gap in the Perovskite $\mathrm{CH}_{3} \mathrm{NH}_{3} \mathrm{PbI}_{3}$. ACS Photon. 2016, 3, 1060-1068.

38. Even, J.; Pedesseau, L.; Katan, C. Analysis of Multivalley and Multibandgap Absorption and Enhancement of Free Carriers Related to Exciton Screening in Hybrid Perovskites. J. Phys. Chem. C 2014, 118, 11566-11572.

39. Zhu, H.; Miyata, K.; Fu, Y.; Wang, J.; Joshi, P. P.; Niesner, D.; Williams, K. W.; Jin, S.; Zhu, X. -Y. Screening in Crystalline Liquids Protects Energetic Carriers in Hybrid Perovskites. Science 2016, 353, 1409-1413. 
40. Bakulin, A. A.; Selig, O.; Bakker, H. J.; Rezus, Y. L. A.; Müller, C.; Glaser, T.; Lovrincic, R.; Sun, Z.; Chen, Z.; Walsh, A.; Frost, J. M.; Jansen, T. L. C. Real-Time Observation of Organic Cation Reorientation in Methylammonium Lead Iodide Perovskites. J. Phys. Chem. Lett. 2015, 6, 3663-3669.

41. Zhu, X. -Y.; Podzorov, V. Charge Carriers in Hybrid OrganicInorganic Lead Halide Perovskites Might Be Protected as Large Polarons. J. Phys. Chem. Lett. 2015, 6, 4758-4761.

42. March, S. A.; Clegg, C.; Riley, D. B.; Webber, D.; Hill, I. G.; Hall, K. C. Simultaneous Observation of Free and Defect-bound Excitons in $\mathrm{CH}_{3} \mathrm{NH}_{3} \mathrm{PbI}_{3}$ using Four-wave Mixing Spectroscopy. Sci. Rep. 2016, 6, 39139.

43. Diab, H.; Trippé-Allard, G.; Lédée, F.; Jemli, K.; Vilar, C.; Bouchez, G.; Jacques, V. L. R.; Tejeda, A.; Even, J.; Lauret, J.; Deleporte, E.; Garrot, D. Narrow Linewidth Excitonic Emission in OrganicInorganic Lead Iodide Perovskite Single Crystals. J. Phys. Chem. Lett. 2016, 7, 5093-5100.

44. Prokhorenko, V. I.; Halpin, A.; Miller, R. J. D. Coherently-controlled Two-dimensional Photon Echo Electronic Spectroscopy. Opt. Express 2009, 17, 9764-9779.

45. Zigmantas, D.; Read, E. L.; Mančal, T.; Brixner, T.; Gardiner, A. T.; Cogdell, R. J.; Fleming, G. R. Two-dimensional Electronic Spectroscopy of the B800-B820 Light-harvesting Complex. Proc. Nat. Am. Soc. 2006, 103, 12672-12677. 
46. Romero, E.; Augulis, R.; Novoderezhkin, V. I.; Ferretti, M.; Thieme, J.; Zigmantas, D.; Grondelle, R. van. Quantum Coherence in Photosynthesis for Efficient Solar-energy Conversion. Nat. Phys. 2014, 10, 676-682.

47. Fuller, F. D.; Pan, J.; Gelzinis, A.; Butkus, V.; Senlik, S. S.; Wilcox, D. E.; Yocum, C. F.; Valkunas, L.; Abramavicius, D.; Ogilvie, J. P. Vibronic Coherence in Oxygenic Photosynthesis. Nat. Chem. 2014, 6, 706-711.

48. Duan, H.-G.; Stevens, A. L.; Nalbach, P.; Thorwart, M.; Prokhorenko, V. I.; Miller, R. J. D. Two-Dimensional Electronic Spectroscopy of Light-Harvesting Complex II at Ambient Temperature: A Joint Experimental and Theoretical Study. J. Phys. Chem. B 2015, 119, 1201712027.

49. Bakulin, A. A.; Morgan, S. E.; Kehoe, T. B.; Wilson, M. W. B.; Chin, A. W.; Zigmantas, D.; Egorova, D.; Rao, A. Real-time Observation of Multiexcitonic States in Ultrafast Singlet Fission using Coherent 2D Electronic Spectroscopy. Nat. Chem. 2016, 8, 16-23.

50. Lim, J.; Paleček, D.; Caycedo-Soler, F.; Lincoln, C. N.; Prior, J.; Berlepsch, H. von; Huelga, S. F.; Plenio, M. B.; Zigmantas, D.; Hauer, J. Vibronic Origin of Long-lived Coherence in an Artificial Molecular Light Harvester. Nat. Comm. 2015, 6, 7755.

51. Elliott, R. J. Intensity of Optical Absorption by Excitons. Phys. Rev. 1957, 108, 1384-1389.

52. Yang, Y.; Yang, M.; Zhu, K.; Johnson, J. C.; Berry, J. J.; van de Lagemaat, J.; Beard, M. C. Large Polarization-dependent Exciton Optical Stark Effect in Lead Iodide Perovskites. Nat. Comm. 2016, 7, 12613. 
53. Saba, M.; Cadelano, M.; Marongiu, D.; Chen, F.; Sarritzu, V.; Sestu, N.; Figus, C.; Aresti, M.; Piras, R.; Lehmann, A. G.; Cannas, C.; Musinu, A.; Quochi, F.; Mura, A.; Bongiovanni, G. Correlated Electronhole Plasma in Organometal Perovskites. Nat. Comm. 2014, 5, 5049.

54. Halpin, A.; Johnson, P. J. M.; Tempelaar, R.; Murphy, R. S.; Knoester, J.; Jansen, T. L. C.; Miller, R. J. D. Two-dimensional Spectroscopy of a Molecular Dimer Unveils the Effects of Vibronic Coupling on Exciton Coherences. Nat. Chem. 2014, 6, 196-201.

55. Chan, W.; Ligges, M.; Zhu, X. -Y. The Energy Barrier in Singlet Fission can be Overcome through Coherent Coupling and Entropic Gain. Nat. Chem. 2012, 4, 840-845.

56. Prokhorenko, V. I. Global Analysis of Multi-dimensional Experimental Data. European Photochemistry Association Newsletter; June 2012, p 21.

57. Butkus, V.; Zigmantas, D.; Valkunas, L.; Abramavicius, D. Vibrational vs. Electronic Coherences in 2D Spectrum of Molecular Systems. Chem. Phys. Lett. 2012, 545, 40-43.

58. Egorova, D. Self-Analysis of Coherent Oscillations in Time-Resolved Optical Signals. J. Phys. Chem. A 2014, 118, 10259-10267.

59. Xie, L. -Q.; Zhang, T. -Y.; Chen, L.; Guo, N.; Wang, Y.; Liu, G.; Wang, J.; Zhou, J.; Yan, J.; Zhao, Y.; Mao, B.; Tiana, Z. Organicinorganic Interactions of Single Crystalline Organolead Halide Perovskites Studied by Raman Spectroscopy. Phys. Chem. Chem. Phys. 2016, 18, 18112-18118.

60. Brivio, F.; Frost, J. M.; kelton, J. M.; Jackson, A. J.; Weber, O. J.; Weller, M. T.; Goni, A. R.; Leguy, A. M. A.; Barnes, P. R. F.; Walsh, A. Lattice Dynamics and Vibrational Spectra of the 
Orthorhombic, Tetragonal, and Cubic Phases of Methylammonium Lead Iodide. Phys. Rev. B 2015, $92,144308$.

61. Park, B. -W.; Jain, S. M.; Zhang, X.; Hagfeldt, A.; Boschloo, G.; Edvinsson, T. Resonance Raman and Excitation Energy Dependent Charge Transfer Mechanism in Halide-Substituted Hybrid Perovskite Solar Cells. ACS Nano 2015, 9, 2088-2101.

62. Glaser, T.; Müller, C.; Sendner, M.; Krekeler, C.; Semonin, O. E.; Hull, T. D.; Yaffe, O.; Owen, J. S.; Kowalsky, W.; Pucci, A.; Lovrinčić, R. Infrared Spectroscopic Study of Vibrational Modes in Methylammonium Lead Halide Perovskites. J. Phys. Chem. Lett. 2015, 6, 29132918.

63. Prokhorenko, V. I.; Picchiotti, A.; Maneshi, S.; Miller, R. J. D. Two-dimensional and Transient Absorption Spectroscopies of Single-stranded DNA in the Deep UV. Springer Proc. Phys. 2015, 162, 432-435.

Acknowledgements This work was supported by the Max Planck Society and the Excellence Cluster "The Hamburg Center for Ultrafast Imaging - Structure, Dynamics and Control of Matter at the Atomic Scale" of the Deutsche Forschungsgemeinschaft. H-G. D. acknowledges financial support by the Joachim-HertzStiftung Hamburg within a PIER fellowship. The authors thank V. I. Prokhorenko for help with the 2D setup and providing 2D data analysis software. Helpful discussion with Dr. Jyotishman Dasgupta, TIFR is also acknowledged. We would also like to acknowledge the help from Khalid Siddiqui for the low-temperature measurement.

Competing Interests The authors declare that they have no competing financial interests. 
Correspondence Correspondence and requests for materials should be addressed to R.J.D.M. (email: dwayne.miller@mpsd.mpg.de) 
(a)

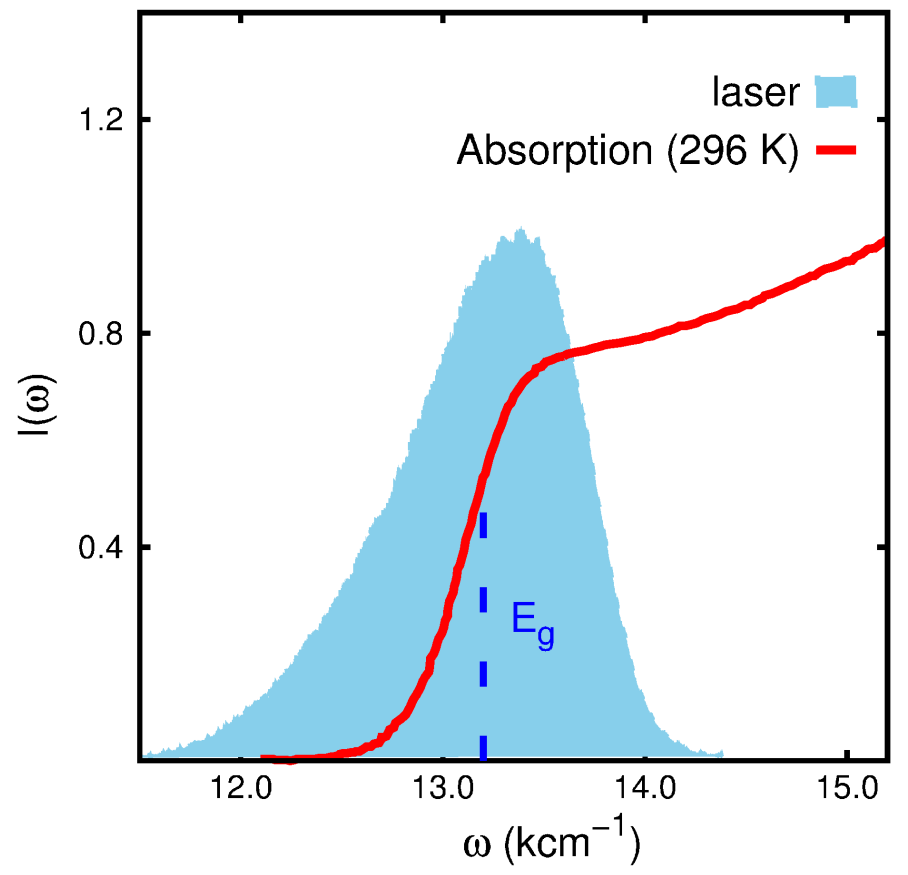

(b)

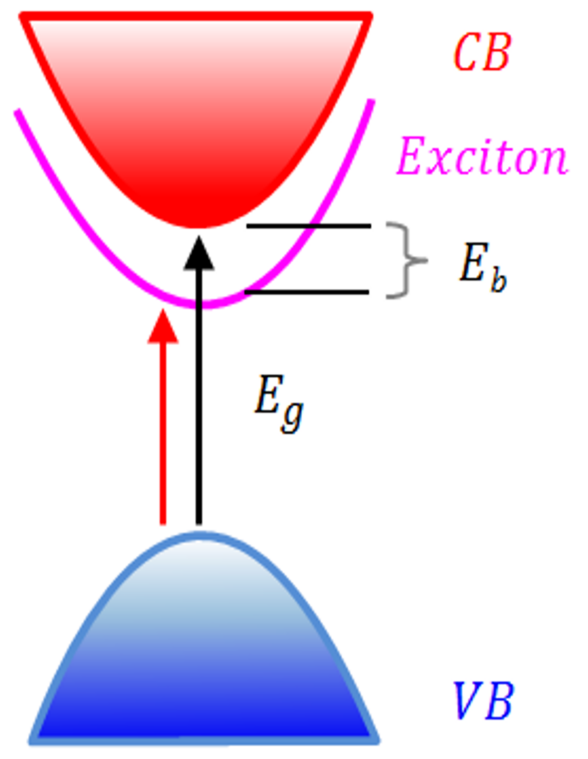

Figure 1: (a) Absorption spectrum of $\mathrm{CH}_{3} \mathrm{NH}_{3} \mathrm{PbI}_{3}$ thin film at room temperature $(296 \mathrm{~K})$ is shown as red trace. The laser spectrum used for 2DES measurements is depicted by the blue shaded curve. The bandgap energy, $E_{\mathrm{g}}$ has been marked as blue dotted line at $13200 \mathrm{~cm}^{-1}$. (b) Free-energy diagram depicting sub-bandgap exciton levels in relation to the conduction band edge. The energy gap between conduction band (CB) minima and exciton level is defined as the exciton binding energy, $E_{\mathrm{b}}$. Black and red arrows represent the bandgap and excitonic transitions, respectively. 

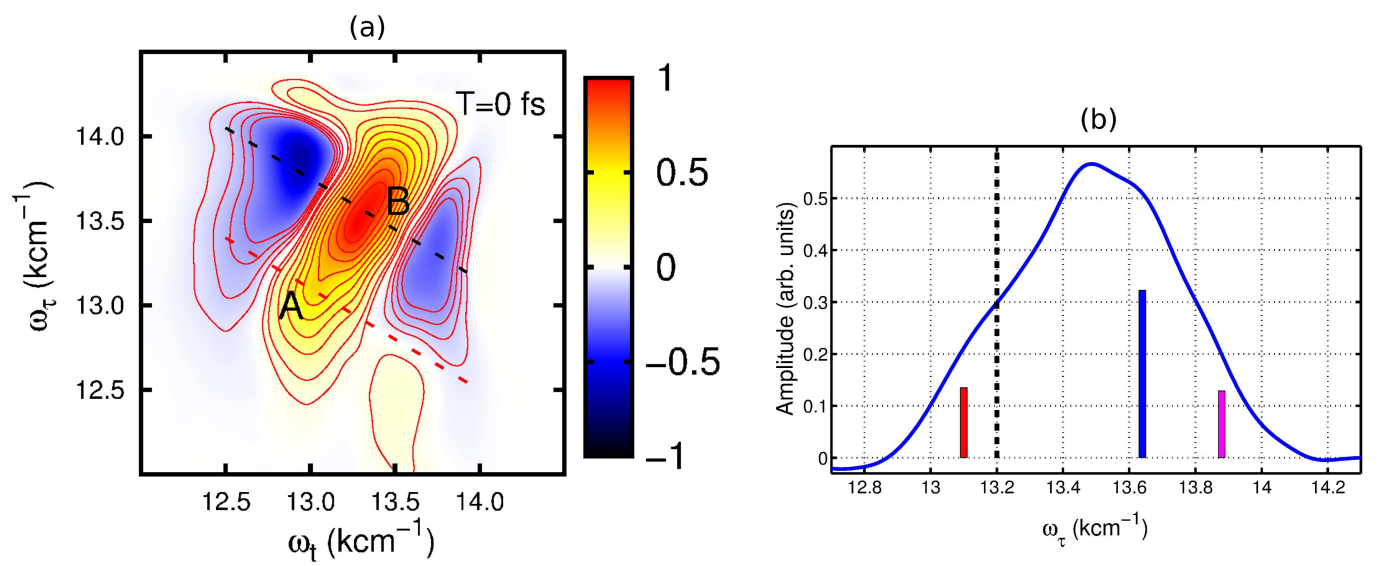

(c)
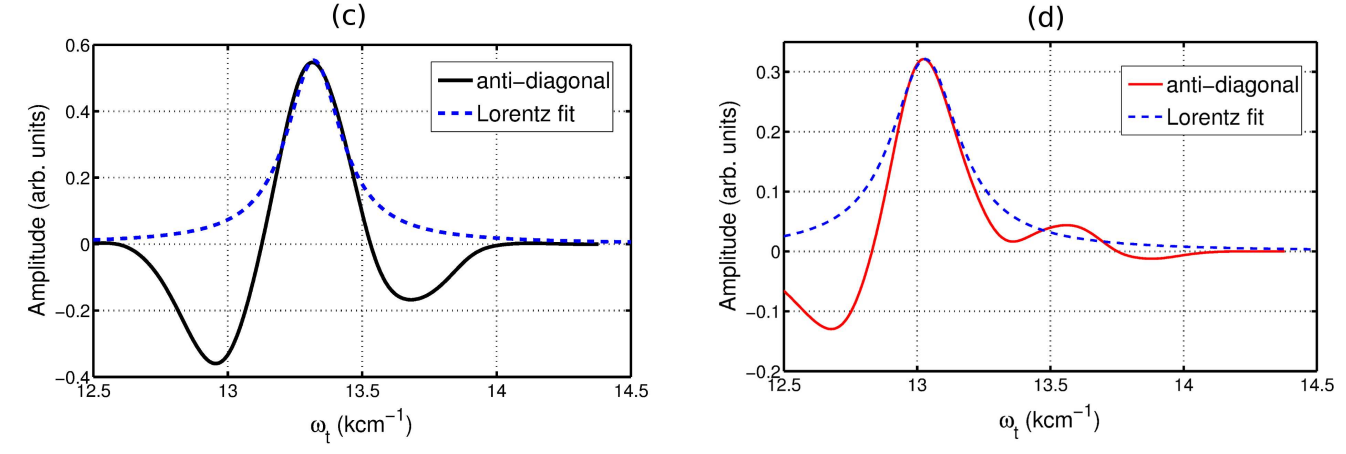

Figure 2: (a) Measured 2D electronic spectrum (total) at room temperature with the waiting time (Red: ground state bleaching, blue: excited state absorption), $T=0$ fs. At $T=0 \mathrm{fs}$, the central peak, B at $\omega_{\tau}=13550 \mathrm{~cm}^{-1}$ is significantly elongated along the diagonal direction, which indicates strong inhomogeneous broadening of the free carriers. An additional central peak has been labeled by A at $\omega_{\tau}=13100 \mathrm{~cm}^{-1}$ which corresponds to the sub-bandgap excitonic transition; (b) a diagonal cutoff from 2D spectrum at $T=0$ fs is projected to $\omega_{\tau}$. In addition to the features in free carrier band (marked by the blue and magenta sticks) above $13500 \mathrm{~cm}^{-1}$, the exciton peak at $13100 \mathrm{~cm}^{-1}$ (marked by the red stick) can clearly be resolved. The dashed line at $13200 \mathrm{~cm}^{-1}$ indicates the bandgap energy for the $\mathrm{CH}_{3} \mathrm{NH}_{3} \mathrm{PbI}_{3}$; (c) and (d) represents the cut along anti-diagonal profile from the 2D electronic spectrum at $T=0 \mathrm{fs}$, which has been marked as (black) and (red) dashed line in (a), respectively. The corresponding bandwidths are resolved by fitting it to a Lorentzian lineshape function, which yields the associated time scales of the electronic dephasing of $41 \mathrm{fs}$ and $47 \mathrm{fs}$ for free carriers in (c) and excitons in (d), respectively. 
(a)

(b)
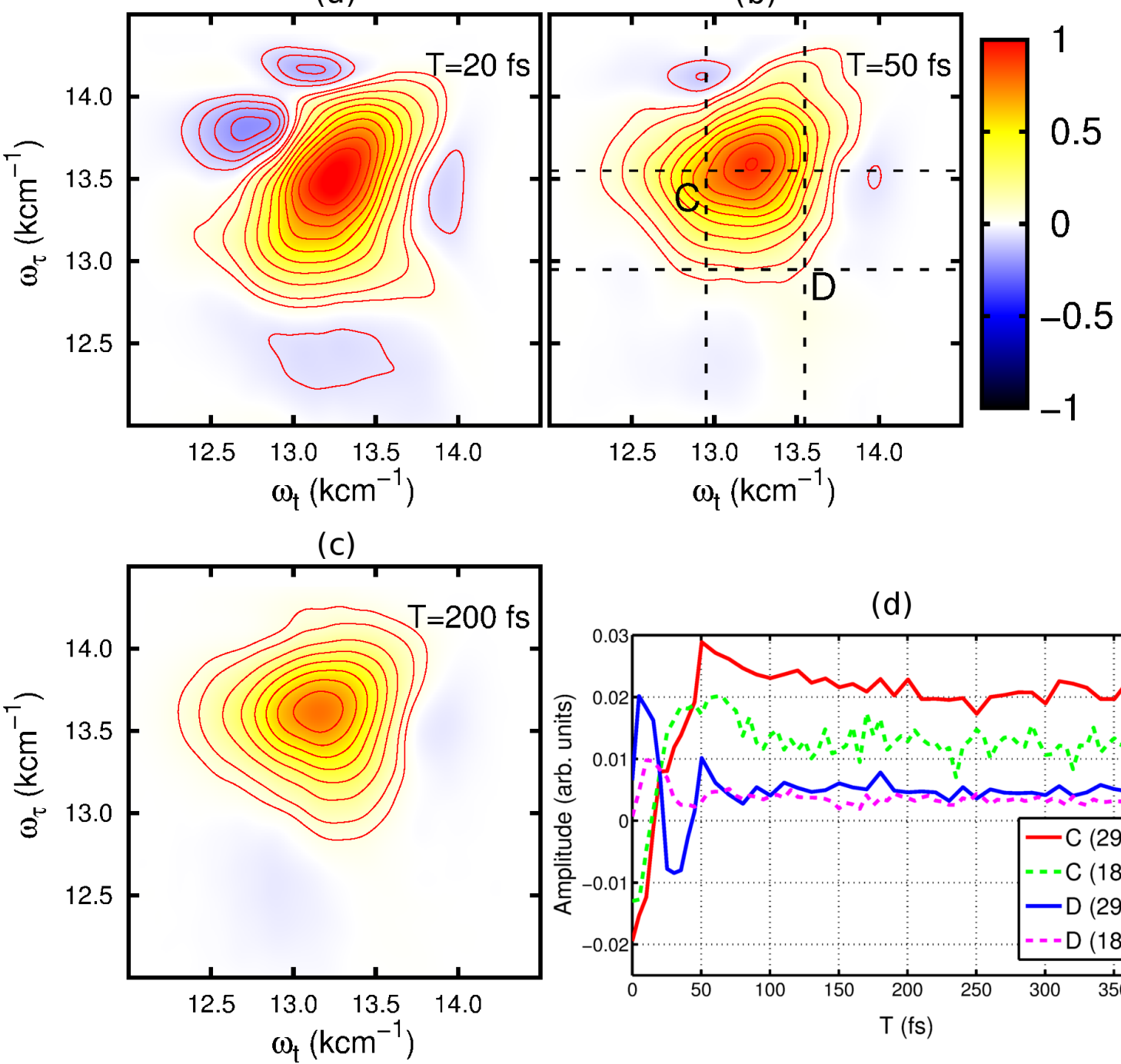

(d)

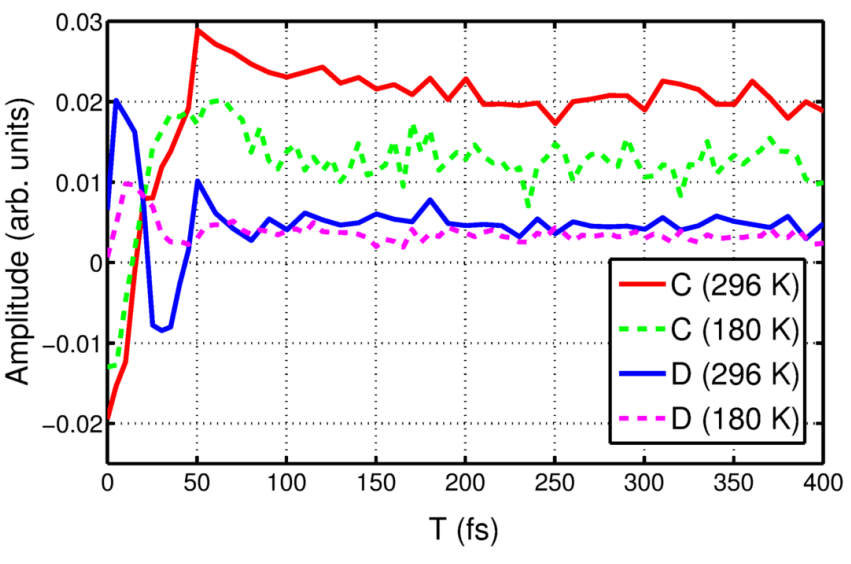

Figure 3: The time-evolving 2D electronic spectra at $20 \mathrm{fs}, 50 \mathrm{fs}$ and $200 \mathrm{fs}$ after photoexcitation are presented in (a), (b) and (c), respectively. The time traces of the cross peaks at $\left(\omega_{\tau}, \omega_{t}\right)=$ $(13550,12950) \mathrm{cm}^{-1}$ and $(12950,13550) \mathrm{cm}^{-1}$ are shown in (d). The corresponding peaks are marked as $\mathrm{C}$ and $\mathrm{D}$ in the $2 \mathrm{D}$ spectrum of $T=50 \mathrm{fs}$. For the comparison, the kinetic traces of cross peaks in $2 \mathrm{D}$ spectra at $180 \mathrm{~K}$ are shown as the dashed lines in green (C) and magenta (D). For the better visualization, the magnitude of the blue line ( $296 \mathrm{~K})$ has been magnified by a factor of 3 . 

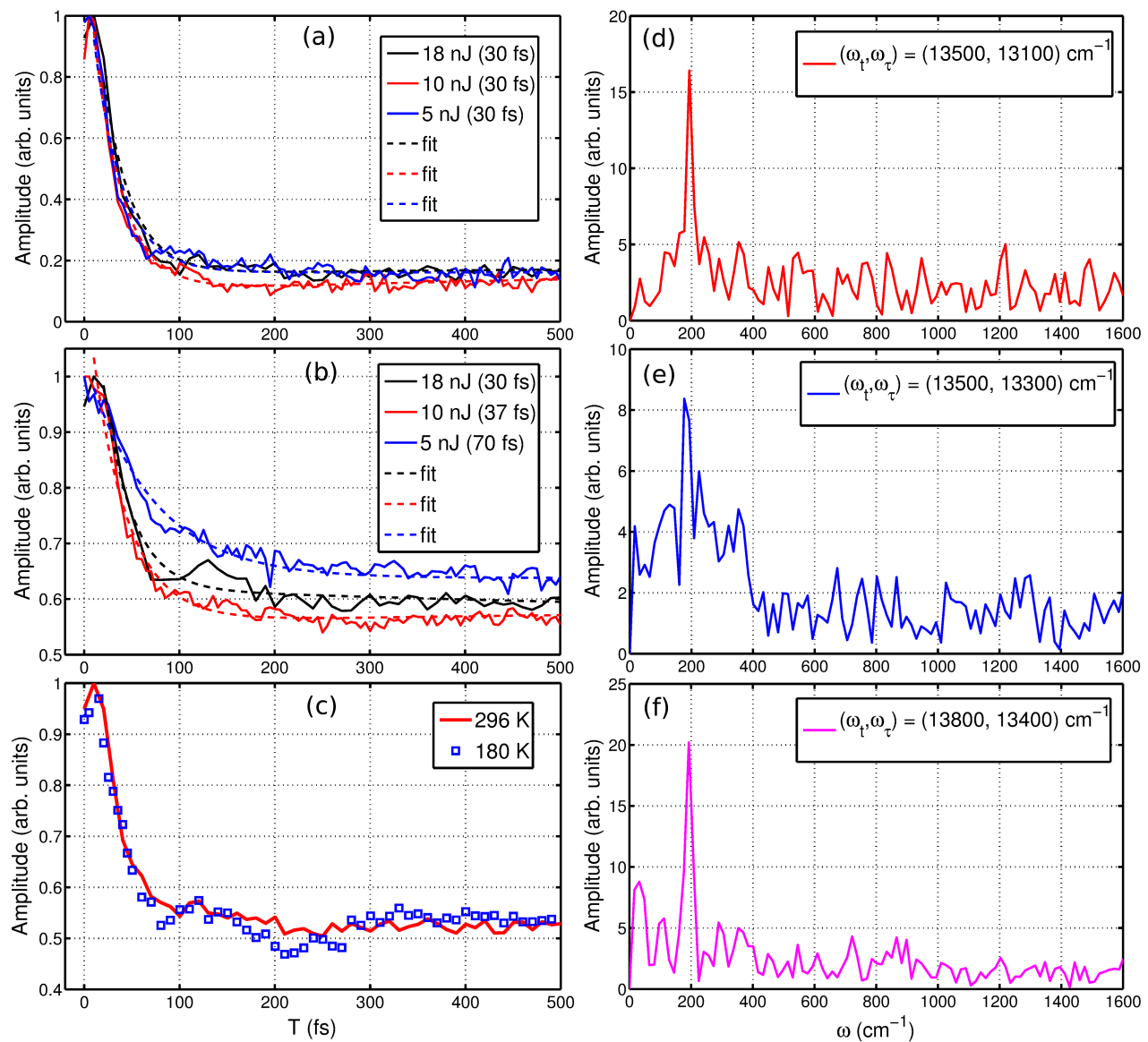

Figure 4: (a) Pump fluence dependence on the exciton and free carrier decay for different excitation energies $(5,10$ and $18 \mathrm{~nJ})$. All the traces show the initial fast exponential decay with the time constant of $30 \mathrm{fs}$. This power-independent exciton dissociation clearly demonstrates the prohibition of exciton-exciton annihilation in our measurement; (b) Dynamics of the free carrier with different excitation energies. The power-dependent decay traces of the free carrier peak show evidence of non-geminate recombination at higher pump-fluence; (c) A comparison of kinetics of the exciton peak, $\left(\omega_{\tau}, \omega_{t}\right)=(13150,13100) \mathrm{cm}^{-1}$ at different temperatures $296 \mathrm{~K}$ (red line) and 180 K (blue square); (d), (e) and (f) The power spectrum of vibrational modes in particular region of 2D spectra. One strong vibrational mode of $190 \mathrm{~cm}^{-1}$ can clearly be observed in all power spectra extracted from different regions of the 2D map. In addition, several low-frequency vibrations can be resolved at $\left(\omega_{\tau}, \omega_{t}\right)=(13500,13300) \mathrm{cm}^{-1}$. The high-frequency modes carry low intensities. 